\title{
Primäre Formen der Hypertriglyzeridämie sind selten, aber gefährlich
}

\author{
Bei Hypertriglyzeridämie sollte man zunächst an eine \\ sekundäre Ursache denken, denn die primären gene- \\ tischen Formen sind sehr selten. Doch letztere kön- \\ nen eine schwere lebensbedrohliche Pankreatitis \\ auslösen, weshalb unabhängig vom kardiovaskulären \\ Risiko eine effektive Therapie erforderlich ist.
}

Bei Hypertriglyzeridämie wird zwischen primären, d.h. genetisch bedingten, und sekundären Formen differenziert. „Primäre Formen sind sehr selten, sodass man zunächst immer an eine sekundäre Ursache denken sollte“, so Dr. Ulrike Schatz, Dresden. Bei der hereditären Hypertriglyzeridämie sind die TG erhöht und HDL-Cholesterin erniedrigt, das LDL-Cholesterin normal. Beim Chylomikronämie-Syndrom sind die TG stark erhöht, HDL- und LDL-Cholesterin erniedrigt. Bei kombinierter Hyperlipoproteinämie sind TG und LDL erhöht, das HDL erniedrigt.

Die meisten Patienten müssen für die Bestimmung der Triglyzeride nicht nüchtern sein. Dies gilt für die Erstbestimmung, für Kinder, beim akuten Koronarsyndrom, bei alten Patienten und bei stabiler medikamentöser Therapie. Eine Nüchtern-Bestimmung ist aber sinnvoll bei Triglyzeriden $>440 \mathrm{mg} / \mathrm{dl}$, bekannter Hypertriglyzeridämie, bei Diabetikern und vor Beginn einer Medikation, die eine starke Hypertriglyzeridämie induzieren kann. Sinnvoll ist auch die Bestimmung des Non-HDL. Dies ergibt sich, wenn man das HDL vom Gesamtcholesterin abzieht. Non-HDL umfasst neben LDL, Lipoprotein (a) und den triglyzeridhaltigen VLDL auch die Remnants. Diese Restpartikel von Chylomikronen sind hochatherogen. Beträgt die Differenz zwischen LDL und Non-HDL $>30 \mathrm{mg} / \mathrm{dl}$, sollte die Therapie intensiviert werden. „Auch wenn in der Leitlinie kein Zielwert für die Triglyzeride angegeben wird, sollte bei Werten $>150 \mathrm{mg} / \mathrm{dl}$ immer nach anderen Risikofaktoren gesucht werden“, so Schatz.

\section{Triglyzeride nur schwacher Risikofaktor} Während erhöhtes LDL und erniedrigtes HDL als eigenständige starke kardiovaskuläre Risikofaktoren gelten, wird der Hypertriglyzeridämie nur eine zusätzliche Bedeutung als solcher zuerkannt. „In einer großen Metaanalyse konnten die Triglyzeride nach Adjustierung für Alter und Geschlecht zwar als kardiovaskulärer Risikofaktor identifiziert werden, aber nach

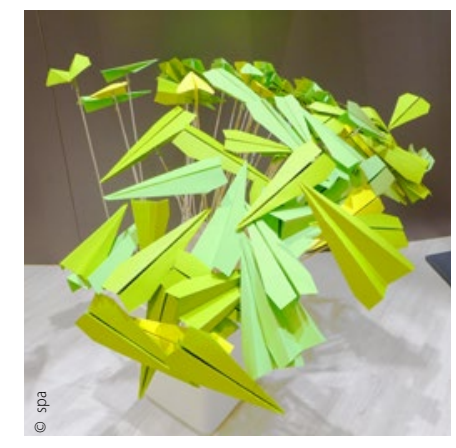

Unterschiedliche Dylipidämien erfordern unterschiedliche Therapien. scheint milchig-weiß und die TG sind meist stark erhöht $(>850$ $\mathrm{mg} / \mathrm{dl})$. Klinisch finden sich bei Betroffenen eruptive Xanthome, eine Hepatosplenomegalie und schwere rezidivierende Pankreatitiden, die nicht selten septisch verlaufen und zu Multiorganversagen führen. Spätkomplikationen sind exkretorische Pankreasinsuffizienz, Diabetes und Pankreaskarzinom. ,Extrem hohe Triglyzeridwerte findet man beim familiären ChylomikronämieSyndrom, das immer als lebensbedrohliche Erkrankung angesehen werden sollte, zumal für diese Erkrankung bisher kaum eine effektive Therapie verfügbar ist", so Schatz. Bei solchen Patienten sei nur der Plasmaaustausch sei eine Option.

Das Spektrum der sekundären Ursachen ist breit. Dazu gehören der Alkoholabusus, die Hypothyreose, das metabolische Syndrom, der Diabetes mellitus, Autoimmunerkrankungen, Schwangerschaft und Medikamente, nämlich Glukokortikoide, Cyclosporin, Östrogene, Tamoxifen, Betablocker, Thiazide, Spironolacton, HIV-Medikamente und Retinoide.

Die Basis der Therapie sind Lebensstiländerungen. „Vorrangig ist die absolute Alkoholkarenz, denn schon $100 \mathrm{ml}$ Bier führen zu einem Anstieg der Triglyzeride um 160 mg/dl“, so Schatz. Die Kalorienaufnahme sollte sich am Normalgewicht orientieren und v.a. sollte die Zufuhr von gesättigten Fettsäuren eingeschränkt werden. Die Diät sollte $<50 \%$ aus Kohlenhydraten, beim Chylomikronämie-Syndrom gar $<15 \%, 10-20 \%$ aus Eiweiß und $<30$ $\%$ aus Fett bestehen. Empfehlenswert sind $>30 \mathrm{~g}$ Ballaststoffe/ Tag. Dazu kommt vermehrte körperliche Aktivität. „Diät plus Bewegung senken die Werte um ca. 50 \%" so Schatz.

\section{Medikamente können notwendig sein}

Während sich die TG bei sekundären Formen durch Beseitigung der Ursache bzw. Veränderung des Lebensstils oft normalisieren, erfordern primäre Formen meist eine Medikation mit einem Fibrat. Damit können die TG um 30$50 \%$ gesenkt werden. „In einer Sekundärpräventionsstudie wurde mit Bezafibrat der primäre Endpunkt aus tödlichem und nicht tödlichem Herzinfarkt plus plötzlichem Herztod um 9,4\% gesenkt, was aber statistisch nicht signifikant war", so Julius. Bei den Patienten mit Hypertriglyzeridämie habe man in der Studie aber die Gesamtmortalität um 25 \% senken können.

Eine ähnliche Wirkung auf die TG-Werte haben Omega-3-Fettsäuren, Statine und Ezetimib weiterer Adjustierung für HDL und Non-HDL war die Assoziation nicht mehr signifikant", so Prof. Ulrich Julius, Dresden. Doch sei in einer Langzeitstudie die 22-Jahres-Mortalität bei Patienten mit einer schweren Hypertriglyzeridämie um 68 \% höher gewesen als bei jenen mit normalen Werten. Und in der Prove-It TIMI-22-Studie zeigte sich ein erhöhtes Rezidivrisiko für ein kardiovaskuläres Ereignis, wenn die TG $>150 \mathrm{mg} / \mathrm{dl}$ betrugen.

Die häufigsten genetischen Störungen sind der Lipoproteinlipase-Mangel, der familiäre ApoC-II- und der ApoA-V-Mangel. Typischerweise sind bei diesen Erkrankungen auch die Chylomikronen und das VLDL erhöht. Das Plasma ist lipämisch und er- dagegen nur geringen Einfluss. Sie werden in der Leitlinie nur für kardiovaskuläre Hochrisikopatienten empfohlen, also vorrangig bei genetisch bedingter kombinierter Hyperlipoproteinämie. Hier sollte Fenofibrat mit einem Statin kombiniert werden. „Eine solche Kombination erfordert am Anfang wöchentliche Kontrollen von Leber- und Nierenwerten und CK", so Schatz. In der Schwangerschaft sollten keine lipidsenkenden Medikamente verordnet werden.

Dr. med. Peter Stiefelhagen

Quelle: „Hypertriglyzeridäme - was tun?" beim Diabeteskongress, 26.5.2017 in Hamburg 